\title{
Pathological response characteristics and surgical outcome following use of emerging neoadjuvant treatment strategies in lung cancer: a narrative review
}

\author{
Irshad Nabi Soomro \\ Directorate of Cancer and Associated Services, Department of Cellular Pathology, Nottingham University Hospitals NHS Trust, City Hospital \\ Campus, Nottingham, UK \\ Correspondence to: Dr. Irshad Nabi Soomro. Consultant Histopathologist \& Honorary Clinical Associate Professor, Directorate of Cancer and \\ Associated Services, Department of Cellular Pathology, Nottingham University Hospitals NHS Trust, City Hospital Campus, Nottingham NG5 \\ 1PB, UK. Email: Irshad.soomro@nuh.nhs.uk.
}

Objective: To review response characteristics relating to emerging neoadjuvant treatment strategies of non-small cell carcinoma of lung, including analysis of scoring systems developed to assess tumour specific effects.

Background: Rapid progress has been made in recent years with regard to the management of patients with non-small cell lung cancer as a consequence of discovery of relevant oncogenic signalling pathways governing cancer cell growth and metastasis, introduction of immune checkpoint inhibitors in lung cancer therapy, and targeted screening for lung cancer in high-risk populations. Histopathologists have diagnostic involvement in interpretation of cytology, biopsy and resection specimen from treated lung cancer patients. They are at the forefront of molecular testing of tissue samples and PD-L1 scoring in lung cancers. Pathologists assessment of tumour resection specimens facilitates assessment of cellular and stromal effects of those novel and emerging neoadjuvant therapy compounds which can include development of scoring methods to assess tumour response following chemo-radiotherapy and targeted therapy. For example, immunotherapy induced changes can be seen in treated tumours and a scoring system has been designed which includes immune related pathologic response criteria (irPRC).

Methods: Experience of increasing involvement with molecular testing of lung cancers, multi-disciplinary team (MDT) attendance and input from treating oncologists, literature review \& search, attendance at courses/conferences and working in multiple tumour sites are behind the writing this invited review.

Conclusions: Targeted therapies in non-small cell lung carcinomas impact on surgical outcomes and prognosis. Treatment options have expanded and currently include surgery, chemotherapy, radiotherapy, targeted therapy and immune therapy. Histopathologists are being increasingly involved in treatment response evaluation of tumour resection specimens relating to various modalities. They need to familiarize themselves with relevant treatment specific changes. Surgeons and clinicians need to provide detailed information along with the specimen from treated lung cancer patients. This review will focus on pathology related issues arising from advances in lung cancer neoadjuvant treatment.

Keywords: Lung cancer; immune therapy; neoadjuvant therapy

Received: 14 July 2021; Accepted: 17 April 2022; Published: 01 December 2022.

doi: 10.21037/asj-21-66

View this article at: https://dx.doi.org/10.21037/asj-21-66 


\section{Background}

The Nobel prize in medicine 2018 was awarded to James P. Allison and Tasuku Honjo for developing immune checkpoint blockade. Their discoveries added cancer immunotherapy to the expanding list of modalities now utilized to treat lung cancer (1). There has been a sea change in the way a histopathologist deals with biopsies and cytology samples relating to diagnosis of lung cancer.

The diagnosis pathways for small cell carcinoma of lung is well established with lung cancer multi-disciplinary team (MDT) involvement and management by oncology \& radiotherapy teams. Diagnosis of non-small cell carcinoma, in contrast, now initiates a complex chain of events including immunomarkers evaluation with molecular testing regimes. Several druggable molecular alterations have shown survival benefit and improved quality of life in lung cancer patients. In pulmonary adenocarcinoma and non-squamous cell carcinoma, several pathways have been identified which can be disrupted by a number of newly identified agents replacing chemo-radiotherapy. These include EGFR pathway (tyrosine kinase \& BRAF inhibitors), P13K/AKT/mTOR pathway (P13K inhibitor, temsirolimus), RAS-MAPK pathway (multikinase inhibitors \& miR-34a), JAK-STAT pathway (JAK 2 inhibitor \& ruxolitinib), HER3 (U3-1287 \& U31402), checkpoint inhibitors (Immunotherapy) and various combinations of these therapeutic modalities (2). But before these molecular tests are requested, a histopathologist has to make a judgment whether there is enough tissue first for diagnosis, and then marker evaluation, typically immunohistochemistry which require presence of at least 100 cells for programmed death ligand 1 (PD-L1) testing and molecular tests. These tests have exponentially increased over last decade (Figure 1). We present the following article in accordance with the Narrative Review reporting checklist (available at https://asj.amegroups.com/article/ view/10.21037/asj-21-66/rc).

\section{Objectives}

Primary surgical treatment is restricted to a small number of lung cancer patients. Most patients receive initial nonsurgical management. The molecular fingerprinting of non-small cell carcinomas helps in detection of actionable targets relating to disrupting pathways which have been showing in clinical trials to improve patient prognosis. Immunohistochemistry and fluorescence insitu hybridization (FISH) are able to detect changes at the protein level (gene amplification, point mutation, DNA re-arrangement and PD-L1 testing). With the increasing number of druggable gene aberrations being identified, next generation sequencing (NGS) is now replacing single alteration conventional techniques. NGS can simultaneously identify a broad spectrum of genomic alterations including single nucleotide variants (SNV), copy number variants (CNV) and structural re-arrangements (3). This review aims to capture these changes in the management of nonsmall cell carcinoma with special reference to neoadjuvant therapy and the pivotal role of histopathologists responsible for timely provision of these diagnostic requirements but also subsequent tumour and stromal cell response evaluation to these various agents.

\section{Methods}

This review article is based on Medline and Google search utilizing lung cancer, molecular testing, PD-L1, neoadjuvant therapy, pathological response in lung cancers, circulating tumour DNA (ctDNA), immunotherapy, signalling pathways for cell death as key words. Years considered for this review are 2010 onwards. Attending pulmonary pathology club meetings, lung cancer day out sessions in Nottingham, exposure to lung cancer MDTs, writing two articles for the Royal College of Pathologists, experience of increasing involvement with molecular testing in lung cancers, input from treating oncologists, attendance at courses/conferences and working in multiple tumour sites have been influential in writing this review.

\section{Discussion}

The landscape of cancer patient management has seen major advancements in recent years. Lung cancer has witnessed the biggest change with a positive impact on patient prognosis. Diagnosis of non-small carcinoma of lung is a trigger for the lung cancer MDT to embark on staging based on computed tomography (CT) and positron emission tomography (PET) scanning. In addition, if regional lymph nodes show disease activity, attempts are made to obtain tissue for diagnosis and molecular testing. Patients with stage I, stage II \& stage IIIa disease are candidates for surgical intervention provided surgeon is happy with the performance status, lung function tests and favourable cardiology opinion. All patients are candidates for chemotherapy, radiotherapy, targeted therapy and immunotherapy. Every effort is made to identify actionable 


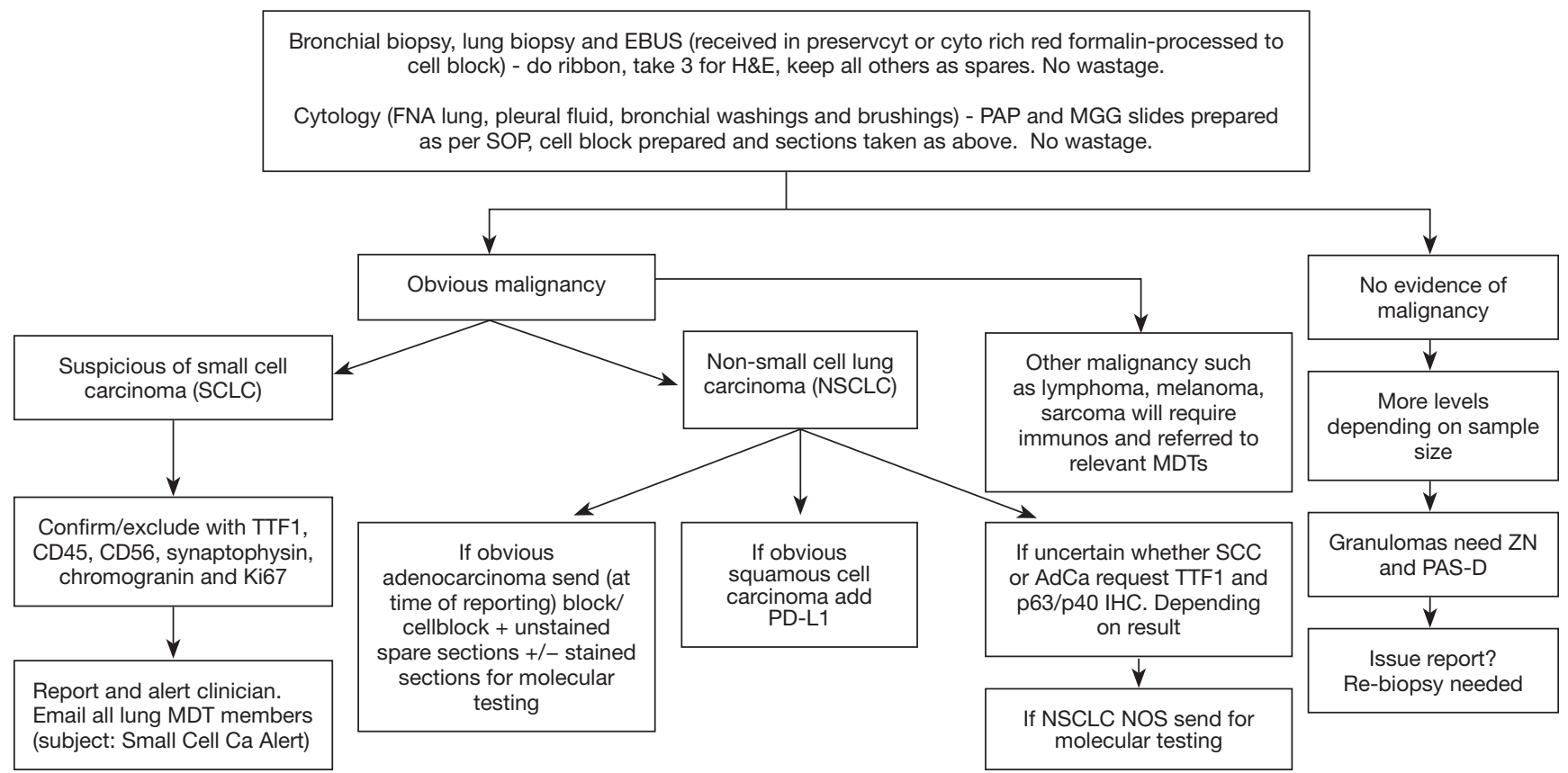

Figure 1 East Midlands Protocol for bronchial biopsies, EBUS, lung biopsies and bronchial cytology. Molecular tests include EGFR, ALK, KRAS, BRAF, HER2, ROS1, RET, MET and NTRK1, NTRK2, NTRK3. PD-L1 is requested through immunolab. Most labs mostly only do $1 \mathrm{H} \& \mathrm{E}$ and reserve all material until next test(s) required. EBUS samples are received either in formalin or cyto rich red. GLH laboratories are doing next-generation sequencing to incorporate most of the molecular markers. EBUS, endobronchial ultrasound; FNA, fine-needle aspiration; PAP, Papanicolaou; MGG, May Grunwald Giemsa; SOP, standard operating procedure; PD-L1, programmed death ligand 1; MDT, multi-disciplinary team; SCC, squamous cell carcinoma; AdCa, adenocarcinoma; IHC, immunohistochemistry; NOS, not otherwise specified; ZN, Ziehl-Neelsen stain; PAS-D, Periodic acid Schiff plus diastase; GLH, genomic laboratory hub; H\&E, hematoxylin and eosin.

mutations/translocations in tumour cells. Adenocarcinoma and non-small cell carcinomas of lung require testing for EGFR mutations, ALK-EML translocations, ROS1, BRAF, MET mutations, RET mutations, HER2 mutations, NTRK1, NTRK 2 and NTRK 3 analysis. The increasing number of biomarkers have highlighted the burden on the notoriously small cell and tissue samples obtained by various diagnostic techniques (4). This can be helped by multiplex laboratory methods particularly use of multiple-biomarker next generation platforms and consolidation of all material into one block. Genomic testing in the NHS is now provided through a national testing network, consolidating and enhancing the existing laboratory provision under the umbrella of NHS genomic medicine service. The national genomic testing service is delivered through a network of seven genomic laboratory hubs (GLHs) each responsible for coordinating services for a particular part of country. This will be the first national health care system to offer whole genome sequencing as a part of routine care. There are seven national genomic hubs and Nottingham is connected to East Genomic Laboratory Hub led by Cambridge University Hospitals NHS foundation trust (5).

This review topic requires familiarisation with terminology used by clinical MDT colleagues. Neoadjuvant therapy encompasses all treatment modalities administered before primary cancer surgical treatment, whereas adjuvant therapy describes regimes administered after primary surgical treatment. There are recognised advantages of neoadjuvant therapy which include less extensive surgery, higher frequency of complete resection, lower rate of pneumonectomy, higher rate of lobectomies/wedge excisions, benefits to management of superior sulcus tumours and enrolment into clinical trials with access to tissue before and after systemic therapy (6).

Lessons can be learnt for use of neoadjuvant therapy and response assessment in other common solid human tumours and the impact on patient prognosis.

Tumour regression grade (TRG) is applied to gastrooesophageal carcinoma patients receiving platinum-based chemotherapy followed by surgery in our institution. In 2009 we described use of the Mandard's grading system 
to assess score for chemotherapy related changes in the tumour (7). Tumours having TRG 1, 2 or 3 were classified as responders to chemotherapy and tumours with TRG 4 or 5 as non-responders. In the neoadjuvant chemotherapy (NAC) group over $45 \%$ of lower oesophageal adenocarcinomas had TRG 1,2 or 3 compared with $13.7 \%$ in the primary surgery group indicating significant tumour down staging. There was positive correlation between TRG and ypTNM staging.

Following this a multicentre study, including our own in Nottingham, to define and validate pathological response to neoadjuvant therapy in oesophago-gastric adenocarcinoma (8) based on questionnaire distributed to $11 \mathrm{UK}$ centres treating gastro-intestinal cancers to determine the use of assessment of response to NAC. Records of patients under-going oesophago-gastric resection at seven centres were reviewed. Pathological response was assessed using Mandard's TRG and lymph node down staging. There was a significant difference in survival between responders (TRG 1-2) and non-responders (TRG 3-5). Among non-responders, the presence of lymph node down staging was associated with significantly improved overall survival compared with patients without lymph node down-staging.

High grade serous carcinoma is the most common subtype of ovarian and fallopian tube carcinoma. Neoadjuvant chemotherapy followed by de-bulking surgery is the treatment of choice. Available treatment options include PARP inhibitors, hormone receptor modulators, immune checkpoint inhibitors and therapeutic vaccines. A six-tiered system subsequently refined to a three tiered system, Chemotherapy response score (CRS) is used for assessment of chemotherapy response. The CRS stratifies patients into complete (CRS 3), partial (CRS 2) and no (CRS 1) response after treatment with various agents (9). CRS categories have significant differences in progression free and overall survival.

Locally advanced oesophageal, gastric and colorectal carcinomas are initially treated by neoadjuvant therapy followed by resection. Histological findings after therapy include residual tumour, inflammation, resorptive changes with infiltrate of foamy macrophages, foreign body reaction and fibrosis. The tumour regression grading system is used in most centres in United Kingdom. TRG refers to presence of residual viable tumour and amount of therapy induced fibrosis. TRG categorisation provides information on prognosis and effects of therapy (10).

A meta-analysis from MD Anderson Cancer Centre has shown that residual cancer burden in breast cancer after NAC can predict disease recurrence and survival across all breast cancer sub-types. This study (11) of 5,160 patients from 12 Institutions examined the relationship between the continuous residual cancer burden index and eventfree survival/distant relapse in four cancer phenotypes. These include triple negative cancers, HER2 positive and Hormone receptor negative, Hormone receptor positive and HER2 negative and Hormone receptor positive and HER2 positive cancers. There was up to $69 \%$ rate of complete pathologic response in HER2 positive hormone receptor negative cases. Lower response rates were seen in hormone receptor positive and HER2 negative cases 11\%, triple negative group $43 \%$ and the dual hormone receptor positive and HER2 positive group 38\% response rate. They suggest that the introduction of synoptic reporting for a post-neoadjuvant response will help keep pace with precision medicine.

In a recently published meta-analysis (12), found 52 articles with patient number totalling 27,895 who had received neo-adjuvant chemotherapy. Patients with a pathologic complete response after neo-adjuvant chemotherapy had significantly better event free survival, particularly for triple negative and HER2+ tumours

A further study in 2018 by Choi et al. (13) evaluated the tumour volume changes in breast before and after NAC. They utilized dynamic contrast-enhanced (DCE) magnetic resonance imaging parameters assessed using a commercially available computer aided system. They concluded that the tumour volume changes in breast cancers before and after neoadjuvant therapy might be more accurate tool for evaluation of the pathologic response after neoadjuvant therapy.

Pathological response in malignant melanomas is observed after neoadjuvant targeted and immune checkpoint therapy. Two patterns of response are observed including hyalinized fibrosis and tumoral melanosis pattern (14). Complete absence of viable tumour cells in the treated tumour bed is deemed pathological complete response (pCR). Major pathological response is defined as less than $10 \%$ of viable tumour cells and partial response as less than $50 \%$ of the treated tumour bed being occupied by viable tumour cells. Patients given neoadjuvant-targeted and immune-checkpoint therapies in melanomas have a correlation with improved relapse free survival.

\section{Pathological response after neoadjuvant treatment in lung cancers}

Lung cancer occupies a slightly different position when 
compared to other solid cancers due to the low surgical resection rate of small cell and non-small cell carcinoma. Data published by Cancer Research UK-Lung statistics, show that only $2 \%$ of small cell carcinomas and $16 \%$ of nonsmall cell carcinomas have surgical resection as primary cancer treatment. This is much lower rate of resection in comparison to other sites. Almost $82 \%$ breast cancer patients have resection of cancer after diagnosis (all stage combined). Resection rate is likely to increase with the introduction of lung cancer screening in high-risk population in United Kingdom with higher chance of picking up low stage disease (15). Also, the British thoracic society guidelines for the management of pulmonary nodules and malignancy prediction calculator (16) with nodule MDT separate from lung cancer MDT are going to have a positive effect on surgical resection rate. Up to $42 \%$ of small cell carcinoma and $27 \%$ of non-small cell carcinomas patients have radiotherapy as part of their treatment. Stereotactic ablative radiotherapy (SABR) for peripheral tumours has delivered better overall survival. Up to $68 \%$ of patients diagnosed with small cell carcinoma and 25\% patients with non-small cell lung cancer have chemotherapy. A smaller number of patients have genomic alterations such as EGFR mutations, ROS1, ALK-EML, BRAF, MET, RET \& NTRK alterations and receive appropriate drugs such as tyrosine kinase inhibitors. Second line agents such as third generation tyrosine kinase inhibitors are reserved for those who progress or develop resistance (17). Patients with no detectable mutations have the option of immune check inhibitor immunotherapy depending upon the extent of expression of PD-L1. Even fewer tumours are receiving neo-adjuvant chemotherapy before surgery. This provides little opportunity to pathologist to assess pathological response to neo-adjuvant therapy in resected lung cancers.

There was a proposal to use major pathologic response as a surrogate endpoint in a paper published in 2016 from Sloan-Kettering Cancer Centre (18). Residual viable tumour cells less than or equal to $10 \%$ following neoadjuvant chemotherapy was deemed a major pathologic response which would potentially meet the criteria for a surrogate. This study aimed to identify associations with improved survival and they proposed that major pathologic response should be used as a surrogate end point for survival in future trials for resectable lung cancers.

Philipp Zens conducted a study on 117 patients with non-small cell lung cancer resected after neoadjuvant treatment between 2000 and 2016 (19). A novel prognostic score (PRSC) combining T-category, lymph node status and major pathologic response were assessed in all patients. The isolated ypT-category and the combined TNM8 stages accurately differentiated overall survival and disease-free survival. Tumour regression had prognostic impact. Optimal cut-offs for major pathological response emerged as $65 \%$ for adenocarcinoma and 10\% for non-adenocarcinoma. The novel prognostic score was helpful in overall survival and disease-free survival.

The most recent and comprehensive paper has been published by Travis et al. in fournal of Thoracic Oncology (20). They have come up with 10 recommendations related to processing of lung cancer resection specimen with a view to define pathologic response, including major pathologic response and complete pathologic response. This review article has provided guidelines on how to recognize the tumour bed, histological assessment of primary tumour and determine pathologic response to neoadjuvant therapy. This group has also commented that it is extremely difficult to distinguish histologic changes related to therapy from those that may have been present without therapy.

Some studies have tried to assess the effects of adjuvant therapy on resected positive lymph nodes (21). Pathologic response in lymph nodes alone or in combination with the primary tumour is able to predict the clinical outcome in treated lung cancers.

The Royal College of pathologist minimum data set for lung cancer reporting recommend an estimation of whether more or less than $10 \%$ residual viable tumour is present in the resection specimen and this should be reported. Letter $y$ should be added before the TNM stage. Complete response would be classified as ypT0 (22).

Histopathologists plays a key role in multidisciplinary management of lung cancer patients. Once a biopsy is received from suspected lung cancer, a diagnosis is made. Tumour sample adequacy is assessed with a view to feasibility of immunohistochemistry and molecular testing. A patient may be deemed suitable for neoadjuvant chemotherapy. This may be followed by surgical resection and assessment of remaining viable tumour. This requires experience in distinction between various types of tissue response variables. There are several signalling pathways of cell death. Two most important are apoptosis and necroptosis. Apoptosis results in activation of cysteine proteases triggering cell death seen as nuclear condensation and membrane blebbing. Necroptosis is a more recently defined lytic form of programmed cell death. This requires activation of receptor-interacting protein kinases RIPK1 and RIPK3 (23). Necroptotic lysis releases many 

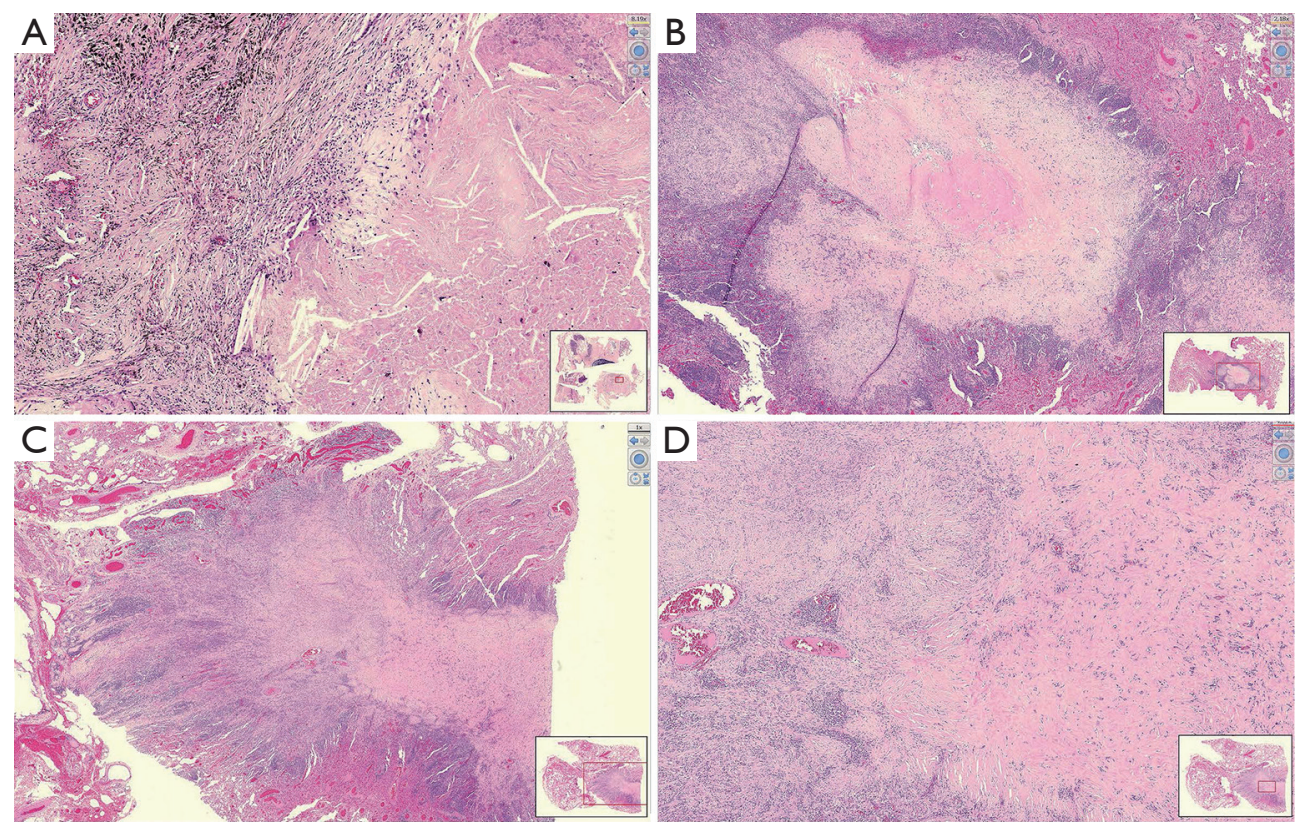

Figure 2 (A) This section shows a focus of complete response to chemotherapy before surgery for non-small cell carcinoma. There is a focus of necrosis surrounded by fibrosis with foreign body giant cell reaction. No viable tumour cells are present. Magnification: $\times 8.19$. (B) A focus of hyalinised fibrosis with a necrotic centre is seen. There is a rim of collection of mature lymphocytes present. No viable tumour cells are seen due to complete response to chemotherapy. Magnification: $\times 2.18$. (C) A focus of fibrosis without necrosis and any viable tumour cells is seen. Magnification: $\times 1.00$. (D) More mature type fibrosis and chronic inflammation seen representing completely treated non-small cell carcinoma. Magnification: $\times 4.02$. Hematoxylin and eosin-stained.

immunogenic damage associated molecular patterns (DAMPs). These can stimulate innate immune pattern recognition receptors (PRRs). Morphological changes after chemotherapy, radiotherapy and targeted therapy do not have any distinguishing features visible by microscopy (Figure 2). Hence it is not possible to distinguish between apoptosis or necroptosis. Visible changes highlighting cell death in epithelial component include nuclear enlargement, nuclear shrinkage, necrosis, vacuolation of nucleus, vacuolation of cytoplasm, dyscohesion, shrinkage of tumour cells with features of non-viability like karyorrhexis, karyolysis and pyknosis. Various agents target stroma as well as the epithelial component. Hence stroma may show fibrosis, elastosis, collagenisation, hyalinization, microcalcification and neovascularization, mucinous change and fibrinoid necrosis. Blood vessels may be hyalinised. The host inflammatory response could be lymphocytic, plasmacytic, histiocytic and giant cell (24). Immune therapy related necrosis mainly manifests as sudden death of the whole cell nest caused by the destruction of frame work of stroma that provides nutrition for the tumour cells. Chemo- radiotherapy causes cell damage by direct toxicity to tumour cell DNA. Neoadjuvant immunotherapy activates tumour specific $\mathrm{T}$ cells and destroys cancer cells.

Three histological patterns following neoadjuvant antiPD-1 therapy treatment have been described which include immune activated (tumour regression caused by immune cells), immune excluded (immune cells contained in the stroma surrounding tumour cells) and immune desert (absence of immune cells in either the parenchyma or the stroma of the tumour) (25). In patients treated with immunotherapy followed by resection, it is important to look at the regression bed which contains lymphocytes, macrophages, tumour cell death-cholesterol clefts and tissue repair-neovascularization with fibrosis. Wound healing/ tissue repair type process has been identified in tumours treated by immunotherapy which is not seen in cancers treated by conventional chemo-radio therapy, likely due to different mechanism of action of immunotherapeutic agents. Immune related pathologic response criteria (irPRC) has been developed which is reproducible amongst histopathologists. This is focussed on regression bed 
having immune activation [(I) dense tumour infiltrating lymphocytes with macrophages and tertiary lymphoid structures; (II) massive tumour death-cholesterol clefts and (III) tissue repair-neovascularization and proliferating fibrosis]. Each feature enriched in major pathologic responders versus nonresponders. Histopathologic features of the regression bed have been used to develop immunerelated Pathologic Response Criteria (irPRC). irPRC could be useful tool to standardize pathologic assessment of immunotherapy (26). Residual viable tumour volume for pathological evaluation of immunotherapy patients may not be sufficient. The histological phenotype of residual viable tumour immune response should be evaluated. This is likely to help guide choice of immunotherapy, chemotherapy or radiotherapy. Overall neoadjuvant therapy is systemic and will affect occult systemic disease, pathological response of the primary tumour may be a predictor of long-term outcomes, has shorter trial timeliness and has ability to assess sensitivity and resistance of adjuvant agents (27).

Another development in recent years is liquid biopsy. Between $15 \%$ and $50 \%$ of patients with stage I through stage III have detectable circulating tumour cells in the peripheral blood in various cancers. ctDNA is present in $50 \%$ to $95 \%$ of patients with stage I through stage III. ctDNA changes after neo-adjuvant therapy and before surgery may have prognostic significance in lung cancers similar to other cancer sites such as rectal cancer. Liquid biopsy has an important role in the overall management of lung cancer patients. Liquid biopsy can also be an important diagnostic tool and can be useful in screening for lung cancer by picking up circulating tumour cells, free DNA, microRNAs, exosomes, antibodies and proteins (28). Pan cancer comprehensive genomic profiling (CGP) for solid tumours from blood and tissue biopsy samples can detect relevant DNA \& RNA variants for multiple types of cancers. Key biomarker for lung cancer detection is AKT1 (Illumina).

\section{Summary}

Various methods have been proposed to measure the response of tumour cells to neo-adjuvant therapies including chemo/radiotherapy, targeted therapies to molecular alterations and immune modulators. Ultimate aim of all these therapies is to induce cell death to eradicate cancer and improve patient prognosis. Our understanding of cell death and signalling pathways has recently been enhanced. This should add value to our assessment of tumours resected with and without any therapy. However, this is still work in progress and requires further input from biologists, pathologists and wide range of scientific community members. Amongst various systems developed in multiple tumour sites, three tier systems are the most popular methods amongst pathologists and clinical teams based on studies demonstrating their relationship to prognosis. Histopathologists receiving resection specimen after immune-therapy treatment need to familiarize themselves with changes caused by neoadjuvant immunotherapy and the updated scoring systems. New modalities to treat cancers will continue to be developed and histopathologists will have a significant role in assessment of tumour response potential and help with assessment of prognosis after treatment. Morphological damage caused by new treatment options can be seen under the microscope and scanned slides. Genotypic damage is difficult to visualize with microscope. NGS will help in evaluation of lung cancer at a molecular level. Therefore, liquid biopsy will have role in screening, diagnosis, molecular testing, assessing response to various treatments and predicting recurrence of treated lung cancers. Surgeons and interventional radiologists/physicians should provide up to date information on treatment provided to patient before surgery. This information is key for appropriate assessment of specimens. Clinical trials and histopathological grading of tumour response are two important tools which help in allocation of resources to various therapeutic agents. In future pathologists are likely to see more resections from lung cancer patients who have been treated with an ever-increasing range of therapeutic modalities. Accurate pathological assessment of tumour response and down-staging by these treatment methods will have an increasingly important role to play in management of patients with non-small cell lung cancer.

\section{Acknowledgments}

I would like to thank Maria Birch, Associate Practitioner Translational Research/Photography Queen's Medical Centre Nottingham University Hospital NHS Trust for help with photography; Dr. Antonia Barbieri, Consultant Pathologist, Department of Cellular Pathology, Northamption General Hospital NHS Trust, Cliftonville, Northamption NN1 5BD for help in developing biopsy protocol; Michelle Ridgwell, Secretary Team 1 Histopathology Department, Nottingham City Hospitals for help with typing Figure 1; and Professor Ian Ogilvie Ellis, Professor of Cancer Pathology, Nottingham University Hospital for his help in proofreading this article. 
Funding: None.

\section{Footnote}

Provenance and Peer Review: This article was commissioned by the Guest Editors (Dragana Jovanovic and Semra Bilaceroglu) for the series "Impact of Novel Neoadjuvant Treatment on Surgery Outcomes in Lung Cancer" published in AME Surgical fournal. The article has undergone external peer review.

Reporting Checklist: The author has completed the Narrative Review reporting checklist. Available at https://asj. amegroups.com/article/view/10.21037/asj-21-66/rc

Peer Review File: Available at https://asj.amegroups.com/ article/view/10.21037/asj-21-66/prf

Conflicts of Interest: The author has completed the ICMJE uniform disclosure form (available at https://asj.amegroups. com/article/view/10.21037/asj-21-66/coif). The series "Impact of Novel Neoadjuvant Treatment on Surgery Outcomes in Lung Cancer" was commissioned by the editorial office without any funding or sponsorship. The author has no other conflicts of interest to declare.

Etbical Statement: The author is accountable for all aspects of the work in ensuring that questions related to the accuracy or integrity of any part of the work are appropriately investigated and resolved.

Open Access Statement: This is an Open Access article distributed in accordance with the Creative Commons AttributionNonCommercial-NoDerivs 4.0 International License (CC BY-NC-ND 4.0), which permits the non-commercial replication and distribution of the article with the strict proviso that no changes or edits are made and the original work is properly cited (including links to both the formal publication through the relevant DOI and the license). See: https:// creativecommons.org/licenses/by-nc-nd/4.0/.

\section{References}

1. Zang X. 2018 Nobel Prize in medicine awarded to cancer immunotherapy: Immune checkpoint blockade - A personal account. Genes Dis 2018;5:302-3.

2. Yuan M, Huang LL, Chen JH, et al. The emerging treatment landscape of targeted therapy in non-small-cell lung cancer. Signal Transduct Target Ther 2019;4:61.

3. Malone ER, Oliva M, Sabatini PJB, et al. Molecular profiling for precision cancer therapies. Genome Med 2020;12:8.

4. Soomro I. Editorial-Lung cancer diagnostics and prognostics- Tissue is an issue. Annals of Medical Case Reports 2017;1:1004.

5. NHS Genomic medicine service website Home>Genomics>NHS Genomics medicine service. Available online: https://www.england.nhs.uk/contact-us/ privacy-notice/nhs-genomic-medicine-service/

6. Bunn PA Jr, Schenk E, Pacheco J, et al. New Developments in Neoadjuvant Therapy for Lung Cancer. Oncology (Williston Park) 2019;33:101-6, 109.

7. Fareed KR, Ilyas M, Kaye PV, et al. Tumour regression grade (TRG) analyses in patients with resectable gastro-oesophageal adenocarcinomas treated with platinum-based neoadjuvant chemotherapy. Histopathology 2009;55:399-406.

8. Noble F, Lloyd MA, Turkington R, et al. Multicentre cohort study to define and validate pathological assessment of response to neoadjuvant therapy in oesophagogastric adenocarcinoma. Br J Surg 2017;104:1816-28.

9. Cohen PA, Powell A, Böhm S, et al. Pathological chemotherapy response score is prognostic in tubo-ovarian high grade serous carcinoma: A systematic review and meta-analysis of individual patient data. Gynecol Oncol 2019;154:441-8.

10. Langer $\mathrm{R}$, Becker $\mathrm{K}$. Tumor regression grading of gastrointestinal cancers after neoadjuvant therapy. Virchows Arch 2018;472:175-86.

11. Helwick C, Symmans WF. Residual cancer burden is prognostic of outcomes across breast cancer subtypes. The ASCO Post 2020;25:1-5.

12. Spring LM, Fell G, Arfe A, et al. Pathologic Complete Response after Neoadjuvant Chemotherapy and Impact on Breast Cancer Recurrence and Survival: A Comprehensive Meta-analysis. Clin Cancer Res 2020;26:2838-48.

13. Choi WJ, Kim WK, Shin HJ, et al. Evaluation of the Tumor Response After Neoadjuvant Chemotherapy in Breast Cancer Patients: Correlation Between Dynamic Contrast-enhanced Magnetic Resonance Imaging and Pathologic Tumor Cellularity. Clin Breast Cancer 2018;18:e115-21.

14. Tetzlaff MT, Messina JL, Stein JE, et al. Pathological assessment of resection specimens after neoadjuvant therapy for metastatic melanoma. Ann Oncol 2018;29:1861-8.

15. Grover H, Ross T, Fuller E. Implementation of targeted 
screening for lung cancer in a high-risk population within routine NHS practice using low-dose computed tomography. Thorax 2020;75:348-50.

16. Callister ME, Baldwin DR, Akram AR, et al. British Thoracic Society guidelines for the investigation and management of pulmonary nodules. Thorax 2015;70 Suppl 2:ii1-ii54.

17. Neal RD, Sun F, Emery JD, et al. Lung cancer. BMJ 2019;365:11725.

18. Hellmann MD, Chaft JE, William WN Jr, et al. Pathological response after neoadjuvant chemotherapy in resectable non-small-cell lung cancers: proposal for the use of major pathological response as a surrogate endpoint. Lancet Oncol 2014;15:e42-50.

19. Zens P, Bello C, Scherz A, et al. A prognostic score for non-small cell lung cancer resected after neoadjuvant therapy in comparison with the tumor-node-metastases classification and major pathological response. Mod Pathol 2021;34:1333-44.

20. Travis WD, Dacic S, Wistuba I, et al. IASLC Multidisciplinary Recommendations for Pathologic Assessment of Lung Cancer Resection Specimens After Neoadjuvant Therapy. J Thorac Oncol 2020;15:709-40.

21. Pataer A, Weissferdt A, Vaporciyan AA, et al. Evaluation of Pathologic Response in Lymph Nodes of Patients With Lung Cancer Receiving Neoadjuvant Chemotherapy. J

doi: 10.21037/asj-21-66

Cite this article as: Soomro IN. Pathological response characteristics and surgical outcome following use of emerging neoadjuvant treatment strategies in lung cancer: a narrative review. AME Surg J 2022;2:35.
Thorac Oncol 2021;16:1289-97.

22. Nicholson AG, Kerr K, Gosney J. Standards and datasets for reporting cancers. Dataset for histopathological reporting of Lung cancers. The Royal College of Pathologists, September 2018:1-46.

23. Messmer MN, Snyder AG, Oberst A. Comparing the effects of different cell death programs in tumor progression and immunotherapy. Cell Death Differ 2019;26:115-29.

24. Sethi D, Sen R, Parshad S, et al. Histopathologic changes following neoadjuvant chemotherapy in various malignancies. Int J Appl Basic Med Res 2012;2:111-6.

25. Ling Y, Li N, Li L, et al. Different pathologic responses to neoadjuvant anti-PD-1 in primary squamous lung cancer and regional lymph nodes. NPJ Precis Oncol 2020;4:32.

26. Cottrell TR, Thompson ED, Forde PM, et al. Pathologic features of response to neoadjuvant anti-PD-1 in resected non-small-cell lung carcinoma: a proposal for quantitative immune-related pathologic response criteria (irPRC). Ann Oncol 2018;29:1853-60.

27. Blumenthal GM, Bunn PA Jr, Chaft JE, et al. Current Status and Future Perspectives on Neoadjuvant Therapy in Lung Cancer. J Thorac Oncol 2018;13:1818-31.

28. Hofman P. Liquid biopsy for lung cancer screening: Usefulness of circulating tumor cells and other circulating blood biomarkers. Cancer Cytopathol 2021;129:341-6. 